\title{
Replication Efficiency of Influenza A Virus H9N2: A Comparative Analysis Between Different Origin Cell Types
}

\author{
Nima Zarrin Lebas ${ }^{1}$, Shahla Shahsavandi ${ }^{1, *}$, Ashraf Mohammadi ${ }^{1}$, Mohammad Majid \\ Ebrahimi ${ }^{1}$, Mehran Bakhshesh ${ }^{1}$ \\ ${ }^{1}$ Razi Vaccine and Serum Research Institute, Karaj, IR Iran \\ *Corresponding author: Shahla Shahsavandi, Razi Vaccine and Serum Research Institute, Karaj, IR Iran. Tel: +98-2634570038, Fax:+98-264552194, E-mail: shahsavandi_s@yahoo.com.
}

Received: October 14, 2012; Revised: November 28, 2012; Accepted: December 9, 2012

\begin{abstract}
Background:Efficient isolation and detection of low pathogenic avian influenza viruses from surveillance samples continues to be a high priority. Currently, the new cell lines are considered for supporting the replication to high virus strains titers.

Objectives: The replication efficiency of a low pathogenic avian influenza virus in different origin cells was evaluated under different conditions.

Materials and Methods: Chicken embryo fibroblast (CEF) cell and human alveolar epithelial cell line (A549) were infected with H9N2 at a multiplicity of infection of 0.1. The amount of infectious virus released into the cell culture supernatants at various post-infection time intervals were tittered by tissue culture infectious dose (TCID50) assay. The impact of these cells adaptation was investigated by determination the virus genes nucleotide sequences.

Results: The influenza virus infectivity was not significant difference in these cells in the presence of trypsin. The results of fusion assay and determination of cellular protease confirmed that A549 cells support virus entry with or without supplemental trypsin. However, the H9N2 virus showed lower titer and infectivity in the trypsin-free infected A549 cells within longer time. The comparative sequence analysis indicated several simultaneously nucleotide substitutions were occurred in NA of the virus replicated in A549 cells resulted in two fixed amino acid changes at positions G320 to A and G414 to A up to the fifth passage.

Conclusions: After seven consecutive passages of both cell cultures, the H9N2 virus showed similar antigenicity, also no change on viral titer level and virus replication behavior in adaptation was found. The results highlighted the use of A549 cells for efficient virus isolation.

Keywords: Influenza virus; H9N2 subtype; replication kinetic
\end{abstract}

\section{Background}

Low pathogenic H9N2 avian influenza viruses have caused outbreaks in poultry, resulted in serious economic losses in Asia. The Euroasian viruses grouped in three sub-lineages on the basis of antigenic and genetic properties (1). The direct transmission of H9N2 viruses from avian to human (2) and development of multiple reassortant H9N2 subtypes (3-5) introduce the virus as a potential pandemic candidate by the World Health Organization. From 1990s, many studies have been made to introduce suitable alternatives of eggs for vaccine production and research purposes. Recently, new cell lines are considered for supporting the replication of a wide variety of high virus strains titers $(6,7)$.

Alveolar epithelial cells and macrophages are the main targets for influenza virus (8). Some related factors to either virus or host involve in the efficient viral propagation in the cells. Hemagglutinin (HA), neuraminidase (NA), and nonstructural (NS) genes play important roles in host specificity and virulence of influenza virus by mediating viral entry into the target cells and membrane fusion (9), removing the sialic acid (SA) receptors and facilitating the release of progeny virus particles (10), and suppressing host innate immune responses (11). The identification of virus-host interaction is essential to investigate potential cell culture systems for explanation of virus replication and adaptation. The interaction depends on the ability of the virus to enter host cells followed by specific binding to SA receptors and activation of HA protease processing (12).

Generally avian influenza viruses bind to terminal $\alpha 2,3$ $\mathrm{SA}$ residues whereas human viruses bind to $\alpha 2,6 \mathrm{SA}$. The changes in receptor specificity in H9N2 viruses lead to a higher affinity binding of $\alpha 2-6 \mathrm{Gal}$ and SA found in the human upper respiratory tract (10). Lee et al. (13) have been suggested that H9N2 viruses belonging to G1 sub-lineage, may be better adapted to the human host and replicates efficiently in human alveolar epithelial cell line (A549)

Implication for health policy/practice/research/medical education:

The direct transmissions of low pathogenic avian influenza A viruses to human and development of multiple reassortant strains introduce the viruses as a potential pandemic candidate. High-titre replication of the viruses on cell culture would be provide an opportunity for isolation of the viruses for diagnostic, research, and vaccine production purposes.

Copyright (C) 2013,Ahvaz JundishapurUniversity of Medical Sciences; Published by Kowsar Corp. This is an open-access article distributed under the terms of the Creative Commons Attribution License, which permits unrestricted use, distribution, and reproduction in any medium, provided the original work is properly cited. 
than other H9N2 sub-lineages. Due to the viral tropism, avian influenza viruses reach high titers when grown in chicken-origin cells (14-16). The efficient replication and infectivity of low pathogenic influenza viruses is achieved in the presence of supplemental trypsin or the type II transmembrane serine proteases (TMPRSS) $(14,17)$. In this study we provide cellular model to investigate the replication characteristics of H9N2 virus in either mammalian or avian-origin cells and compared their replication efficiencies in seven subsequent passages. We was examined the supernatants from each passage and determined if any changes occurred within the viral genome sequences.

\section{Methods and Materials}

\subsection{Virus and Cell Cultures}

A high-growth H9N2 virus belongs to G1 sub-lineage (18) was used for infecting the chicken embryo fibroblast (CEF) and A549 cells in this study. CEF cells were prepared from 10-day-old embryonated SPF eggs and maintained in Dulbecco's modified Eagle's medium (DMEM) with 10\% fetal bovine serum (FBS) and antibiotic solution. A549 cells (ATCC CCL-185 ${ }^{\mathrm{TM}}$ ) were cultivated in DMEM medium according to the instruction. Before the infection, expression of viral activating protease (VAP) and TMPRSS2 mRNAs in cell cultures were determined using the onestep RT-PCR mixture (Maxime RT-PCR premix; iNtRON Biotechnology, Korea).

Primers are shown in Table 1. Both proteases were expressed in A549, whereas TMPRSS2 was not detected in the CEF cells. Therefore, A549 cell culture was infected with H9N2 virus at multiplicity of infection (MOI) of 0.1 $\mathrm{PFU} /$ cell in $1 \mathrm{~mL}$ in DMEM containing 10\% FBS, antibiotics solutions with and without supplemental trypsin and CEF cell was infected only in the presence of trypsin. Following adsorption for 1 hour at $37^{\circ} \mathrm{C}$, the inoculum was removed and DMEM replaced. Up to 96 hours postinfection (hpi) and at 16-h intervals, culture supernatants were collected and stored at $-70^{\circ} \mathrm{C}$ until used. The effect of cell passage on virus replication dynamics was determined by seven consecutive passages. In case, supernatant of each passage served as the virus seed for the next passage. The virus growth dynamics were evaluated by virus titration of cell culture supernatants at different hpi by tissue culture infection dose ( TCID $_{50}$ ) assay.

Table 1. The Primer Sequences Used for Amplification of the Studied Genes

\begin{tabular}{lllll}
\hline Gene & Forward Primer & Reverse Primer & Length, bp & Accession, No. \\
\hline $\mathbf{H A}$ & CTCGAGCAAAAGCAGGGGAATTTCT & AAGCTTTTATATACAAATGTTGCACCT & 1761 & FJ794817 \\
$\mathbf{N A}$ & CTCGAGAGCAAAAGCAGGAGTAAAAATG & AAGCTTAGTAGAAACAAGGAGTTTTTT & 1431 & JX456183 \\
$\mathbf{N S}$ & CTCGAGAGCAAAAGCAGGGT- & GGATCCAGTAGAAACAAGGGTGTTTTTA & 890 & JX308782 \\
& GACAAAAAC & & & NM205022 \\
$\mathbf{V A P}$ & TGCTGCTCATTGCATAAACC & GGGACTTCGAGCACTTTCAG & 330 & AF329454 \\
$\mathbf{T M P R S \boldsymbol { 2 }}$ & ATCGACAAATGAGGGCAGAC & GTAGGCTGGGGACACTACCA & 480 & L08165 \\
\hline $\boldsymbol{\beta}$-actin & TGCTGTGTTCCCATCTATCG & TTGGTGACAATACCGTGTTCA & 150 & \\
\hline
\end{tabular}

\subsection{TCID50 Assay}

Aliquots of viral supernatants were 10-fold serially diluted with PBS, applied in each A549 and CEF cells/well of a 96 -well plate, and incubated at $37^{\circ} \mathrm{C}$ for 1 hour. The inoculum was removed, and the cells were washed with PBS and supplied with DMEM containing FBS and trypsin. At seven consecutive passages, the $\mathrm{TCID}_{50}$ was determined based on the Reed-Muench method (19).

\subsection{Antigenicity Test}

To measure the antigenicity of viruses in each cell passage, H9N2 reference antisera (20) was used to measure antibody titers using the standard hemagglutinination inhibition (HI) assay (19).

\subsection{Fusion Assay}

The low-pH treatment was performed on H9N2 infected cells with the reaction buffer (DMEM, pH 5.0) for 5 minutes at $37^{\circ} \mathrm{C}$. The buffer was removed and the cells incubated for 8 hours at $37^{\circ} \mathrm{C}$ in fresh neutral DMEM containing 5\% FBS. Cells were fixed with ethanol and stained with Giemsa solution. Fusion activity was determined by counting the fused cells in an entire field.

\subsection{Virus Genes Nucleotide Sequences}

To investigate the effect of adaptation in CEF and A549 cells, full-length viral HA, NA, and NS genes were detected for passages 2, 4, 6 and 8. The primer sets listed in Table 1 were designed for amplification of the genes. The RT-PCR reaction consisted of 1 cycle at $42^{\circ} \mathrm{C}$ for 60 minutes and $95^{\circ} \mathrm{C}$ for 5 minutes and 30 cycles at $94^{\circ} \mathrm{C}$ for 30 seconds, $57.5^{\circ} \mathrm{C}$ for 45 seconds, and $72^{\circ} \mathrm{C}$ for 1 minute, followed by a final elongation step of $72^{\circ} \mathrm{C}$ for 10 minutes. The purified PCR products of viral genes at different passages were sequenced in both directions.

\section{Results}

The H9N2 virus was well replicated in CEF cell and showed the cytopathic effect (CPE) in the presence of 
trypsin. The CPE was appeared in infected A549 cells with or without trypsin treatment Figure 1.

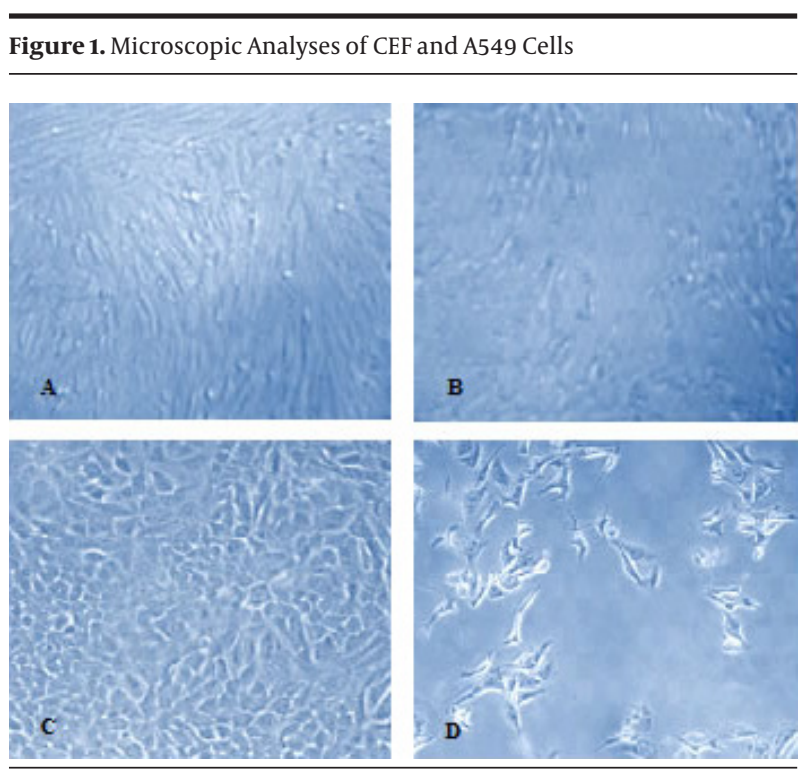

A) mock CEF cell, B) H9N2 infected CEF cells 72hpi, C) mock A549 cell, D) H9N2 infected A549 cells with supplemental trypsin 72 hpi (40x magnification).

The infection of A549 cells in the absence of trypsin recognizes VAP and or TMPRSS2 as HA-processing proteases for the low pathogen subtype virus. The TCID ${ }_{50}$ results showed a decrease in virus titer on the first passage which remained constant during the subsequent passages (Figure 2).

Figure 2. Replication of H9N2 Influenza Virus in Different Cell Origin Types

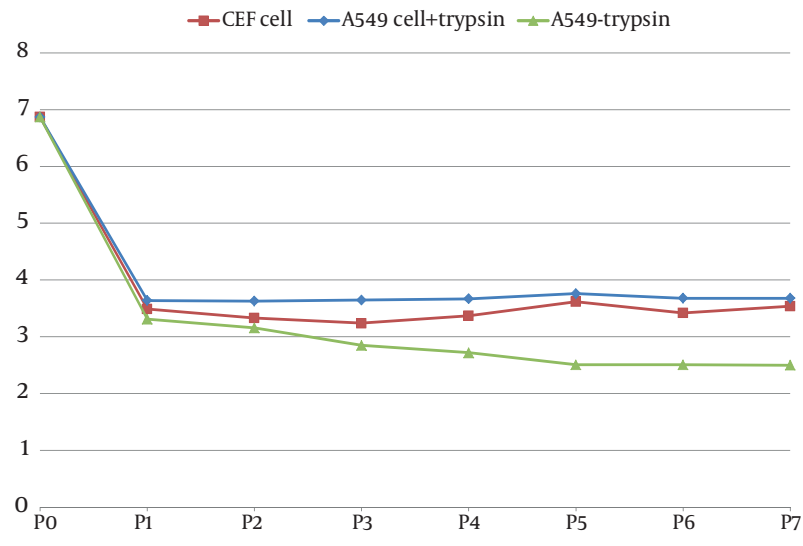

Viral titers of the CEF and A549 infected cells were measured at seven consecutive passages.

The CEF and A549 cells supplemented with trypsin were produced 3.6 and $3.8 \log _{10} \mathrm{TCID}_{50} / \mathrm{mL}$, respectively which were less than the corresponding amount in chicken embryonated eggs (6.7). The H9N2 virus-infected A549 cell culture supplemented with trypsin exhibited the higher virus titer in shorter time compared to the trypsin-free infected cells. The HI results indicate the H9N2 virus in subsequent cell passages have similar antigenicity in both cell cultures. The average number of fused cell per field in A549 cells was not significantly different (46.42 in trypsin-treatment cell vs. 41.35 in non-treatment cell), confirming that the cell supported fusion and virus entry with or without trypsin treatment. The expression of the VAP and TMPRSS2 mRNAs in A549 cells, confirmed the role of cellular proteases in activation of the low pathogenic H9N2 virus.

Nucleotide sequences of the HA, NA, and NS genes of the virus passaged in the cell cultures were analyzed and compared with the parental virus. The comparative sequence analysis indicated several simultaneously nucleotide substitutions resulting in amino acid changes were occurred in NA of the virus replicated in A549 cells. Two fixed amino acid substitutions at positions $\mathrm{G}^{320}$ to $A$ and $\mathrm{G}^{414}$ to A of NA were found up to the sixth passage. The amino acid sequences at the active sites of NA protein at positions 366 IRKDSRAG 373, 399 DSDNRSGY 406, and 431 PQE 433 were conserved. No amino acid changes were observed at the cleavage site, the receptor binding pocket and within the N-glycosylation sites of HA protein. All of them showed conservation of residues $\mathrm{H}^{183}, \mathrm{~L}^{190}, \mathrm{~L}^{226}$, $\mathrm{Q}^{227}$ and $\mathrm{G}^{228}$ in the receptor binding pocket and RSSR motif in cleavage site. All of the viruses possessed the typical avian ESEV motif in PDZ domain at the C-terminal end of NS1 protein. The substitution of amino acid D to $E$ in position 92 which is required for high virulence was not shown.

\section{Discussion}

The replication of enveloped influenza viruses in host cells is a polygenic procedure depends on the host cell endocytic pathways for entry and transfer of viral genome as well as activation of host cell signaling $(21,22)$. The viruses replicate primarily in the epithelial cells of lung and intestines, then spread to the resident macrophages and recruited type II monocytes (23). A variety of cells have been evaluated to investigate the genetic basis of avian influenza viruse hosts. To gain a better understanding of how influenza viruses adapt to the new host, we passaged a high-growth H9N2 influenza strain in two different origin cells and determined replication efficiency of the virus and the molecular basis of adaptation.

The viral titers were decreased in CEF and A549 cells at first passage which remained constant up to the last passage. The final viral titer of $2.2 \log _{10} \mathrm{TCID}_{50} / \mathrm{mL}^{-1}$ was detected in trypsin-free A549 infected cells suggests that under this condition, virus replication was not prevented but clearly delayed. The peak of cell viral titer was accessed after 48 hours. This is in agreement with Sutejo and coworkers (24) suggested that H9N2 virus replicated less efficiently in A549 cell type. The appearance of CPE in A549 cell culture in the absence of trypsin correlates with 
the cleavage of HA protein. Böttcher et al. (12) have been reported that TMPRSS2 expressed in human airways is virus-activating protease that cleaves the monobasic cleavage motif of a H3N2 influenza virus. We show that the protease is able to activate the monobasic cleavage site of the H9N2 virus and support its infection in A549 cells. The A549 cells are permissive to the H9N2 virus growth, but fibroblastic cells that lack the enzymes, require additional trypsin for virus replication $(14,25)$.

The cell fusion was detected in infected A549 cells without supplemental trypsin, therefore; incomplete infection can be a reason for the lower virus titers. Addition of trypsin mediates fast propagation of influenza virus infection and leads to escape viruses from host defense mechanisms (26). The interferon (IFN) response is one of the main host defense mechanisms which expressed during influenza viruse infections. The outcome of IFN induction during influenza virus infection and its suppression by NS1 as well as its effect on virus replication indicate that the antiviral state inhibits virus replication at strain-specific level $(27,28)$. In human cells the replication of the avian influenza viruses is correlated with activation of types I and III IFN and cell-death signaling pathway (24).

A549 cells are IFN-competent and in the absence of trypsin the infection may result in a considerably stronger induction of IFN signaling and apoptosis, which significantly reduced the virus yields. However, the abilities of the cells to support the growth of avian influenza viruses are still debated because of possible change in receptor specificity in avian and the mammalian, susceptibility to higher concentrations of exogenous trypsin, etc. Results of this study demonstrated after seven serial passages of H9N2 virus in the cell cultures, the viral titer was not increased, which is indicating that the avian virus has not adapted to the human tissue culture; even if the viral antigenicity and sequencing patterns of the viral genes were stable.

\section{Acknowledgements}

There is no Acknowledgements.

\section{Authors' Contribution}

None declared.

\section{Financial Disclosure}

There is no Financial Disclosure.

\section{Funding/Support}

The authors have nothing to disclose.

\section{References}

1. Guan Y, Shortridge KF, Krauss S, Chin PS, Dyrting KC, Ellis TM, et al. H9N2 influenza viruses possessing H5N1-like internal genomes continue to circulate in poultry in southeastern China. $J$
Virol. 2000;74(20):9372-80.

2. Butt KM, Smith GJ, Chen H, Zhang LJ, Leung YH, Xu KM, et al. Human infection with an avian H9N2 influenza A virus in Hong Kong in 2003. J Clin Microbiol. 2005;43(11):5760-7.

3. Garcia-Sastre Adolfo, Iqbal Munir, Yaqub Tahir, Reddy Kolli, McCauley John W. Novel Genotypes of H9N2 Influenza A Viruses Isolated from Poultry in Pakistan Containing NS Genes Similar to Highly Pathogenic H7N3 and H5N1 Viruses. PLoS ONE. 2009;4(6).

4. Li C, Yu K, Tian G, Yu D, Liu L, Jing B, et al. Evolution of H9N2 influenza viruses from domestic poultry in Mainland China. Virology. 2005;340(1):70-83.

5. Nagarajan S, Rajukumar K, Tosh C, Ramaswamy V, Purohit K, Saxena $\mathrm{G}$, et al. Isolation and pathotyping of H9N2 avian influenza viruses in Indian poultry. Vet Microbiol. 2009;133(1-2):154-63.

6. Genzel Y, Reichl U. Continuous cell lines as a production system for influenza vaccines. Expert Rev Vaccines. 2009;8(12):1681-92.

7. Jordan I, Vos A, Beilfuss S, Neubert A, Breul S, Sandig V. An avian cell line designed for production of highly attenuated viruses. Vaccine. 2009;27(5):748-56.

8. Nicholls JM, Chan MC, Chan WY, Wong HK, Cheung CY, Kwong DL, et al. Tropism of avian influenza A (H5N1) in the upper and lower respiratory tract. Nat Med. 2007;13(2):147-9.

9. Thompson CI, Barclay WS, Zambon MC, Pickles RJ. Infection of human airway epithelium by human and avian strains of influenza a virus. J Virol. 2006;80(16):8060-8.

10. Geraghty Robert J, Su Bin, Wurtzer Sébastien, Rameix-Welti Marie-Anne, Dwyer Dominic, van der Werf Sylvie, et al. Enhancement of the Influenza A Hemagglutinin (HA)-Mediated Cell-Cell Fusion and Virus Entry by the Viral Neuraminidase (NA). PLoS ONE. 2009;4(12)

11. Hale BG, Randall RE, Ortin J, Jackson D. The multifunctional NS protein of influenza A viruses.J Gen Virol. 2008;89(Pt10):2359-76.

12. Bottcher E, Matrosovich T, Beyerle M, Klenk HD, Garten W, Matrosovich M. Proteolytic activation of influenza viruses by serine proteases TMPRSS2 and HAT from human airway epithelium. JVirol. 2006;80(19):9896-8.

13. Lee Davy CW, Mok Chris KP, Law Anna HY, Peiris Malik, Lau Allan SY. Differential replication of avian influenza H9N2 viruses in human alveolar epithelial A549 cells. Virology Journal. 2010;7(1):71.

14. Shahsavandi Shahla, Ebrahimi Mohammad Majid, Mohammadi Ashraf, Zarrin Lebas Nima. Impact of chicken-origin cells on adaptation of a low pathogenic influenza virus. Cytotechnology. 2012;65(3):419-424.

15. Shen Ching-I, Wang Ching-Ho, Shen Shih-Cheng, Lee Hsiu-Chin Liao Jiunn-Wang, Su Hong-Lin. The infection of chicken tracheal epithelial cells with a H6N1 avian influenza virus. PLOS ONE. 2011;6(5).

16. Zaffuto KM, Estevez CN, Afonso CL. Primary chicken tracheal cell culture system for the study of infection with avian respiratory viruses. Avian Pathol. 2008;37(1):25-31.

17. Chaipan C, Kobasa D, Bertram S, Glowacka I, Steffen I, Tsegaye TS, et al. Proteolytic activation of the 1918 influenza virus hemagglutinin. J Virol. 2009;83(7):3200-11.

18. Shahsavandi S, Salmanian AH, Ghorashi SA, Masoudi S, Ebrahimi MM. Evolutionary characterization of hemagglutinin gene of H9N2 influenza viruses isolated from Asia. Res Vet Sci. 2012;93(1):234-9.

19. World Health Organization . Manual for the Laboratory Diagnosis and Virological Surveillance of Influenza: WHO Global Influenza Surveillance Network:: World Health Organization; 2011.

20. Shahsavandi S, Salmanian AH, Ghorashi SA, Masoudi S, Fotouhi F, Ebrahimi MM. Specific subtyping of influenza A virus using a recombinant hemagglutinin protein expressed in baculovirus. Mol Biol Rep. 2011;38(5):3293-8.

21. Lakadamyali M, Rust MJ, Zhuang X. Endocytosis of influenza viruses. Microbes Infect. 2004;6(10):929-36.

22. Zhang H. Tissue and host tropism of influenza viruses: importance of quantitative analysis. Sci China C Life Sci. 2009;52(12):110110.

23. Herold S, von Wulffen W, Steinmueller M, Pleschka S, Kuziel WA Mack M, et al. Alveolar epithelial cells direct monocyte transepithelial migration upon influenza virus infection: impact of che- 
mokines and adhesion molecules. JImmunol.2006;177(3):1817-24

24. Mossman Karen L, Sutejo Richard, Yeo Dawn S, Myaing Myint Zu, Hui Chen, Xia Jiajia, et al. Activation of Type I and III Interferon Signalling Pathways Occurs in Lung Epithelial Cells Infected with Low Pathogenic Avian Influenza Viruses. PLoS ONE. $2012 ; 7(3)$.

25. Moresco KA, Stallknecht DE, Swayne DE. Evaluation and attempted optimization of avian embryos and cell culture methods for efficient isolation and propagation of low pathogenicity avian influenza viruses. Avian Dis. 2010;54(1 Suppl):622-6.
26. Randall RE, Goodbourn S. Interferons and viruses: an interplay between induction, signalling, antiviral responses and virus countermeasures.J Gen Virol. 2008;89(Pt 1):1-47.

27. Hayman A, Comely S, Lackenby A, Hartgroves LC, Goodbourn S, McCauley JW, et al. NS1 proteins of avian influenza A viruses can act as antagonists of the human alpha/beta interferon response. J Virol. 2007;81(5):2318-27.

28. Wolff T, Ludwig S. Influenza viruses control the vertebrate type I interferon system: factors, mechanisms, and consequences. J Interferon Cytokine Res. 2009;29(9):549-57. 